\title{
A review: Smart Distribution Grid Management using Agents
}

\author{
EHAB M. ESMAIL ${ }^{1}$, MAHMOUD A. ELSADD ${ }^{2}$, NAGY I. ELKALASHY ${ }^{2,3}$, TAMER KAWADY ${ }^{2,4}$ \\ Electrical Engineering Department \\ ${ }^{1}$ Marg High Institute for Engineering and Modern Technology \\ ${ }^{2}$ Faculty of Engineering, Minoufiya University \\ ${ }^{3}$ Faculty of Engineering, Taif University \\ ${ }^{4}$ Faculty of Engineering, Umm Al-Qura University \\ ${ }^{1} 13754$ Marg, ${ }^{2} 32511$ Shebin Elkom, ${ }^{3}$ Taif, ${ }^{4} 421955$ Makkah \\ ${ }^{1,2}$ EGYPT, ${ }^{3,4}$ SAUDI ARABIA \\ 1dr.ehab@mie.edu.eg
}

\begin{abstract}
This paper focuses on the main features of the smart distribution grid (grid management using agents). The fault management is consisting of three stages including faulted section identification, faulted section isolation, and restoration. The probable control strategies utilized in the smart grid are either centralized, decentralized, or autonomous control. Multi-Agent System (MAS) based model uses both centralized and decentralized strategies. The fault management process is usually carried out based on MAS. A literature survey on fault management control schemes is investigated. Furthermore, the reliability is estimated for both centralized, decentralized, autonomous, and modified centralized control strategies. This paper presents reliability modeling and analysis of all considered control strategies. In this paper, comprehensive detailed reliability models of considered control strategies are developed. Using this equivalent reliability model, various reliability indices are calculated.
\end{abstract}

Key-Words: - Smart Grid, Fault management, Multi-Agents; Fault Location Detection, Isolating, and Service Restoration.

Received: March 29, 2020. Revised: July 17, 2020, Accepted: October 14, 2020. Published: November 2, 2020.

\section{Introduction}

Modern communication algorithms use to link all portions of the electric network with each other using sensors and smart devices such as intelligent electronic devices (IEDs) to facilitate self-healing in the smart grid [1-3]. Also, a deregulated distribution network became complex for fulfilling self-healing. Furthermore, enhancing cryptography techniques used for security and privacy protection for the strategies of self-healing for the deregulated distribution networks is in a continuous increasing [3-6]. Such self-healing mechanisms help to realize fully automated fault management [7]. the fault management process starts after disconnecting the feeder and accordingly disappearing the feeder voltage. However, the fault management stages need a control strategy to verify the self-healing perspective. the contemporary control strategies are centralized [8], decentralized [9], autonomous controls [10], and modified centralized control [11] strategies. if the decision is taken at the control center level, the control is called centralized. if the decision is at the secondary substation levels starting from the primary substation, this is decentralized control. if the decision is taken at the secondary substation initiated from the secondary substations level, it is called autonomous control.

For achieving these controls, each substation is implemented by an agent to receive and send the message based on its fault passage indicators (FPIs) status or the output of fault location algorithms. also, reducing both failure index's for communication hops and FPIs or average errors for fault location algorithms utilized to boost the smart grid reliability is still a challenge for researchers.

The main objective of this paper is to have a good understanding of the smart grid features, and agents, through an intensive literature review. A comprehensive literature review of Fault Detection, Isolating, and Service Restoration (FDIR) is introduced. The core focus is on fault management control schemes. 


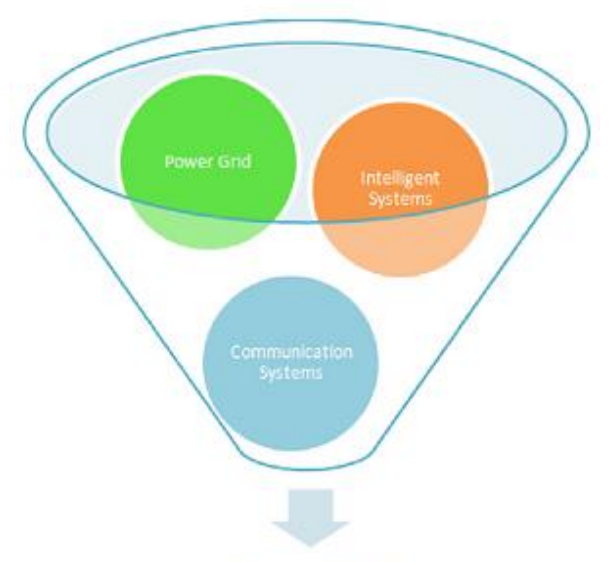

Smart Grid

Fig. 1 Smart grid idea [9].

The review paper is organized into nine sections; section 2 starts the main features of the smart grid. Section 3 focuses on smart grid agents. Section 4 flashes the development of FDIR. Section 5 highlights new fault management and a proposed control strategy. Sections 6,7 , and 8 conclude its reliability for all control strategies at different consideration. Section 9 declares the failure probability of its management.

\section{Smart Grids Features}

Smart grid was integrated transducers technologies, developing control strategies, and modern communication to the power grids to make the complete vision into reality [12]. Fig.1 declares a guide of smart grids technology. As seen in this figure, its grid consists of three parts including power grid, intelligent systems, and communication systems [13]. Distribution generation (DG) was provided many advances including improved reliability, and enhance security as well as inexpensive electricity $[14,15]$. In the smart grid, the impact of faults on customers was minimized by an instantaneous identification of the fault location, correct decisions, system restoration of healthy parts, and recovery of the system to normal condition in $[16,17]$.

Fig. 2 shows a view of the relation between layers in the smart grid and communication network to integrate both in the system [18]. Intelligent Electronic Device (IED) was utilized to control the power flow while making a practical decision [19]. The monitoring and control of the smart grid were enhanced by incorporating the internet in its grid [20]. This requires a modification of the security in the grid to avoid any attacks from hackers. Many types of communication for the transmission media in the smart grid were designed in [21-25].

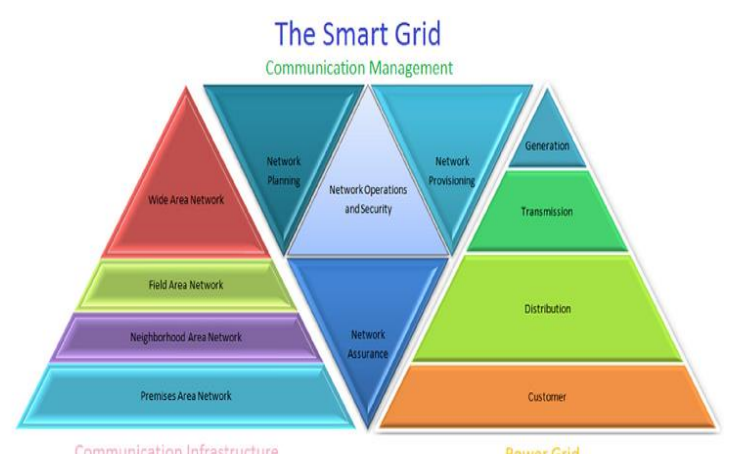

Fig. 2 Communication infrastructure of smart grid [18].
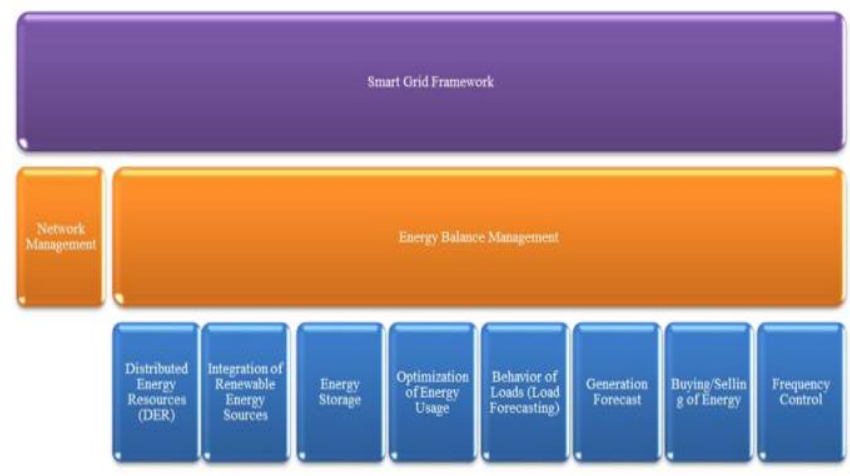

Fig. 3 Features of smart grid [18].

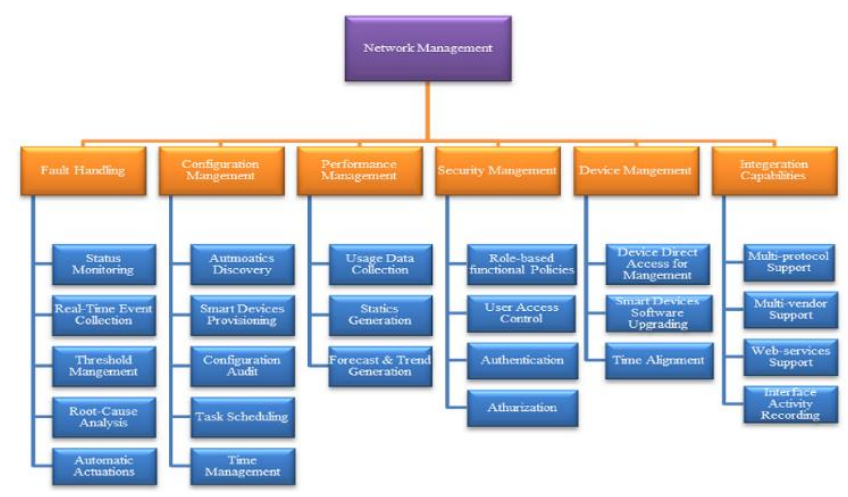

Fig. 4 Network management in the smart grid [28].

A smart grid concentres on the fair pricing and continuity of power supply [19]. In [26], a study for costs and advanced for the smart grid was introduced. Several problems related to the economics of the smart grid were explained in [27]. Figs.3, and 4 declare the smart grid features and the network management in the smart grid, respectively. As seen in Fig.4, the smart grid network management consists of fault handling, configuration management, performance management, security management, device management, and integration capabilities [28]. 


\section{Smart Grid Agents}

The agent system in the smart grid was assisted the reliable and protective operation in a dynamic scenario while acting as centralized control, a distributed control system in [29]. The disturbance diagnosis in [30, 31], service restoration in [32], and secondary voltage control in [33] were proposed. Traditionally Supervisory Control and Data Acquisition (SCADA) in the smart grid was proposed in [34]. The nature of the SCADA system is centralized. This system requires a lot of communication power to make a precise decision for all systems. Consequently, the SCADA system having a much burden on the main process. Hence, its system might be some processing delay. The risk of a big problem in the SCADA system caused by this delay was increased. SCADA system had lacked security. Hence, its system was faced with its issue related to the reliability of the smart grid. In [35], national communication was introduced a detailed report about SCADA. Centralized control demands a complex communication system to collect all data to the control center in the smart grid. It does no matter; the location of the data collection is far from the control center. Therefore; long-distance communication links are required. Despite the large capital investment in the communication, the probability of fault communication link can occur in the system. The control system is become overwhelmed by a big number of tasks if the powerful computer was used.

Phasor Measurement Units (PMUs) had mounted for monitoring. The data from digital relays and PMUs (current, voltage outputs) were used in the SCADA Software to make an overall supervision system. The PMUs data was used to view the power system behavior, which detected abnormal condition [36]. Different communication protocols were used to transfer the information between different devices including I/O level, bay level, substation level, and control center level. All communications were modified using the standard IEC60870 protocol. In the IEC60870 protocol, the fault detection time was improved (decreased) from one hour to 3 minutes. Modern communication protocol (IEC61850) was done between the substations. On the contrary, a conventional protocol can perform peer-to-peer communication for fast messaging between different relays using GOOSE messages. Intelligent Electronic Devices (IED) receive GOOSE messages from other IEDs to take action without the need for centralized control [37]. To achieve the objective of data sharing between different IED at different levels integrated communication infrastructure was used [38]. a)

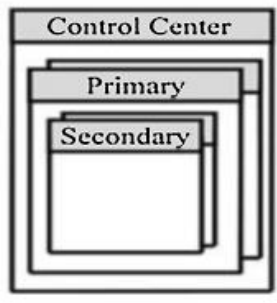

b)

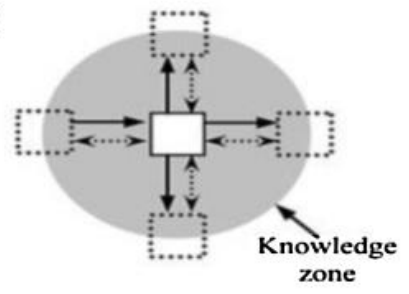

Fig. 5 Control levels and view of the secondary substation object [10].

Fault Passage Indicators (FPIs) were used in different smart grid to collect fault information by using wireless susceptibility to achieve instantaneous action [39].

The communication between FPIs and the control center using GSM was used to reduce the duration of customer interruption by automatically isolating the faulty section and restoring the service for healthy parts without human interference [40].

FPIs and remote-control Ring Main Units (RMUs) were utilized to apply self-healing in the smart grid by using a centralized controller [41, 42]. In $[40,43]$, FPIs were supervised in a smart grid using fiber optics connected to Remote Terminal Units (RTUs) with GSM modem to send any event to the SCADA system.

All recoded events were stored in the database of the SCADA system. Accordingly, the report for all events in the smart grid was done in the control center through TCP/IP and DNP3 protocols. In [44], centralized supervision with emergency local logic control through a SCADA system was proposed. Remote Terminal Units (RTUs) with a Programmable Automation Controller (PAC) were updated based on IEC61850 and DNP3 standard protocols by using Ethernet. Distributed PAC was achieved all $\mathrm{I} / \mathrm{O}$ from the smart grid. In [45], three communication capacities were compared and declared the advantages of IEC61850-based Ethernet using optical fiber for GOOSE in a centralized substation.

To overcome the disadvantages of a centralized control strategy, a Multi-agent system (MAS) was incorporated in the centralized control to achieve decentralized natural $[46-50,10]$. MAS was utilized to simplify a complex problem and then solved them efficiently. This simplicity was done by dividing the problem into sub-problems to perform parallel processing until achieving the main purpose. This parallel processing was reduced the computation burden on a single machine. In [51], decentralized control of the smart grid using agents was introduced. The isolation of the faulty section in the grid was done in agents. 


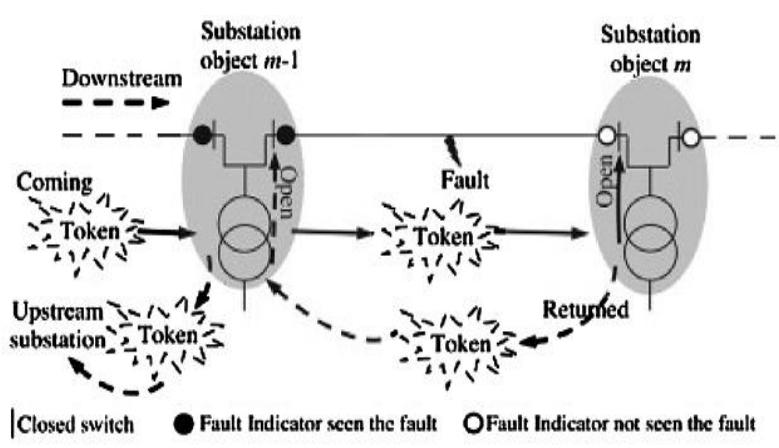

Fig. 6 Decentralized fault management strategy [10].

In [52], [53-59] MAS were developed to facilitate the self-healing features in the smart grid. Figs.5.a, b declare the three-level information hierarchies and the communication between neighboring secondary substations.

As seen in Fig. 6, the example of multi agentsystem control in the smart distribution grid to manage the fault events. In this figure, the fault portion was locally isolated from the healthy part. After the fault has happened between the two neighboring substations, the circuit breaker disconnects the feeder. Consequently, the voltage disappears in all sections and the fault management process is starting. Therefore, the primary substation was sent a downstream token (request data) toward the secondary substations as long as the fault section is identifying.

Fig. 7, declares the multi-agent system in the smart grid. These agents are usually consisting of a three-layer deliberative layer, coordination layer, and reactive layer. The reactive layer performs an instantaneous self-healing action based on programmed knowledge. The coordination layer checks the starting event from the reactive layer based on the predefined priorities. A starting event allows us to reach the top layer only if it exceeds the preset threshold. Deliberative Layer prepares highlevel decisions with the help of the information provided by the coordination layer. The coordination layer in MAS makes the communication between the reactive layer and the deliberative Layer compatible for both. The reactive layer contains many agents that locally control the system behavior and makes short-term planning. Deliberative Layer analyzes the system to make long-term planning [60]. Seven layers of the MAS control hierarchy declare in Fig.8. Several automatic fault location detection and isolation algorithms based on MAS were proposed to enhance the reliability/availability, and efficiency of the smart grid $[52,61]$. All of these techniques survey in the next section.

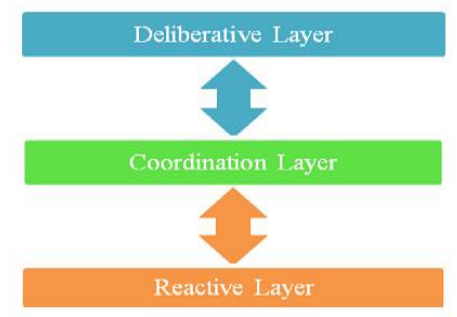

Fig. 7 Three Layers of MAS in the smart grid [60].

\begin{tabular}{|c|}
\hline Main Grid Agents \\
\hline TSO Level Agents \\
\hline Distribution Level Agents \\
\hline Microgrid Agents \\
\hline DER Agents \\
\hline Prosumer Agents \\
\hline Component Level and Load Level Agents \\
\hline
\end{tabular}

Fig. 8 Seven layers of MAS in the smart grid [18].

\section{FDIR Algorithms Based on MAS}

Different methods were implemented to locate the fault in the smart grid such as apparent impedance [62-64]. In [62], the thee-phases circuit analysis was measured such as the local voltage and current measurements in the substation. The fault location algorithms were obtained after iterative solution or complicated three-phase circuit equations. These equations were described as the steady-state fault condition. In $[11,65]$, the earth fault locations were depended on the equality between the computed sequence components of the current at the fault point at different load conditions. Traveling wave methods were discussed in $[66,67]$. These methods are based on the frequency component of traveling waves for faulted voltages.

In [68], the proposed method was calculated as the fault location in the radial smart grid with different laterals. After the fault has occurred, highfrequency transients were generated and propagated outs into both sides. The fault location method was estimated based on the time interval between the traveling waves and the time reflections arriving from the fault location. On the contrary, the traveling wave method was affected by the presence of laterals and load taps [62]. Artificial intelligent based methods such as an artificial neural network (ANN), Fuzzy Logic (FL), Expert System (ES), and hybrid method were discussed in [69-71].

Both methods were used post network status, list of tripped breakers, protection alarms, and the conventional event log. 
In [72], sectionalized re-closure, midpoint reclosure, and tie-point re-closure were integrated with IED for monitoring and protection. The reclosure has detected the fault, isolated the fault section, and restored the power to the healthy part of the smart grid. The re-closure was connected to the grid through a radio or GSM module based on IEC61850 protocol capabilities. Three different FDIR approaches using a loop control scheme, IEC61850 peer-to-peer, and a decentralized controlbased substation were proposed. In [73], the reliability of the network was enhanced by increasing the number of re-closer. The re-closer was communicated by using a high-speed ethernet communication system through fiber optics in the SCADA system. This method was enhanced the restoration time for the healthy section. In [74], advanced communication technology was used to manage the stored information from a smart grid by using IED devices and smart metering. This technology enhances the reliability and starts the self-healing features. Several approaches were proposed in a centralized by including heuristics, expert systems, metaheuristics, and mathematical programming [51]. The expert's knowledge and experience were translated into programming logic to solve the problem in $[75,76]$. In [75], the proposed reconfiguration algorithm was introduced to reduce the loss of service restoration based on switching indices. These indices were provided by using the branch voltage drop, line constants, and weight factors. Integrated load curtailment (LC) for serving customers in the restoration plans was proposed in [76]. This algorithm was based on heuristics rules by using the post-fault power flow calculations. The input information is the number and percentage of the LC candidate which is predetermined. In [77], the service restoration method with a minimum number of switching operations was proposed. Expert system-based techniques were proposed in [78, 79]. This system was involved in the restoration of the Expert's knowledge as rules (IF-THEN statements). In [78], developed an Expert system combined with an objective-oriented programming technique were proposed for solving the restoration issues. Different strategies such a single grouping and multi-grouping were utilized by dividing the outage region into single or multiple groups based on feeder margins, branch points, and available tie switches in [80].

In [81], an Expert system was solved the service restoration problem. This Expert system was constructed a knowledge base system with 180 rules. Group restoration, zone restoration, and load transfer were applied.

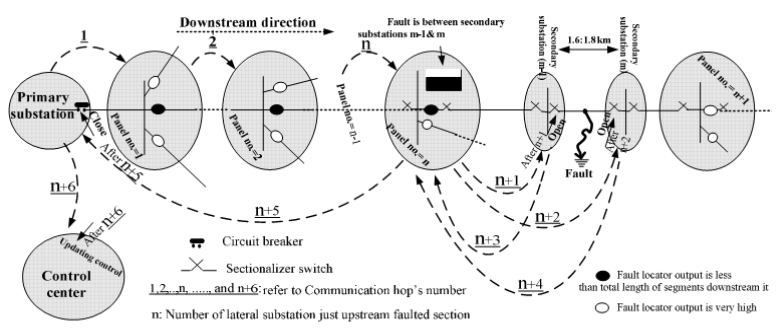

Fig. 9 Modified centralized control strategy [11].

Many researchers were utilized to solve restoration issues [82-84]. This approach such as Neural Network (NN), Genetic Algorithm (GA), FL, Tabu Searching (TS), Practical Swarm Optimization (PSO), Simulated Annealing (SA), and Ant Colonies (AC). In [82], Non-dominated Sorting Genetic Algorithm-II (NSGA-II) was used to solve the service restoration issues. Meta-heuristics approaches require comprehensive computation time when applied for a practical smart grid distribution grid. The mathematical programming approach was determined the target configuration for the restoration plan by formulating its issue Mixed-Integer Non-Linear Problem (MINLP) in $[85,86]$. In [51], a two-layers expert-based MAS framework was developed for monitoring, calculating, and treating during the self-healing. The proposed MAS was designed to restore the out of service. In [87], MAS was utilized to solve the service restoration issue in the smart grid. This strategy doesn't consider the load shedding, customer priorities, or load transfer. Accordingly, the proposed method was faced with the limit of service restoration. In [88], proposed MAS for the restoration problem using two types of agents (Bus Agent (BA), and Facilitator Agent (FA)) was presented. BA was developed to decide a suboptimal configuration after fault happened through integrated with BA based on local data. FA was used to manage the decision process. The restoration process was simplified and completed through a simple BA with the supervision of the FA. In the same paper, the authors don't consider customer priority. In [89], operation and self-healing strategies were proposed in the smart grid with the DG unit.

\section{New Fault Management Strategy \\ 5.1 Modified Centralized Control [11]}

In [11], incorporating fault location application in a centralized fault management strategy was proposed to reduce the measuring devices as seen in Fig. 9. 


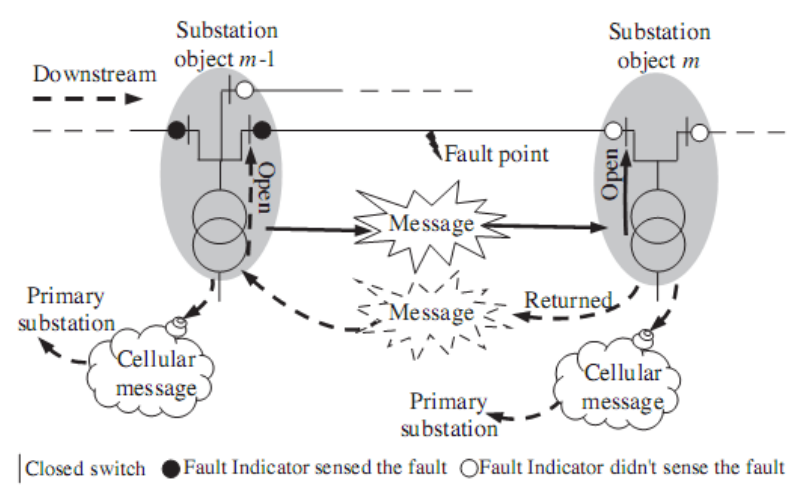

Fig. 10 A Proposed control strategy [10].

Despite the strategy is centralized; the latency is reduced because the communication only between the lateral substations. The fault location algorithm was computed in the lateral substation. After the fault location is calculating, the message from lateral substation was sending to corresponding secondary substations by using the communication between them. Accordingly, the upstream and downstream switches were isolated. The numbers of communication hops are equal to the number of lateral substations forward to the faulty point. This strategy is suffering from false identification for the faulty lateral caused by the error of the fault location.

\subsection{A proposed Control [10]}

The term autonomous is usually using to refer the agent making a decision depends on the prior knowledge gives to its at determining time. An autonomous strategy which greatly used nowadays for the smart grid. Multi-Agent System (MAS) has verified to the most intelligent control strategy entity having distribution control nature. This meaning the fault is resolved locally. The autonomous strategy was preliminarily introduced in [10]. This strategy is advanced by decreasing the latency time due to a lower number of activated communication hops. This strategy is starting when the feeder voltage disappears. All secondary substations before the fault (upstream direction) are sensing the fault using FPI and transmit a signal to the next substation (Downstream direction). If one of the two secondary substations, the downstream substations received a message from the upstream substation indicating to a fault and also its substation did not detect the fault which the fault occurs between them as seen in Fig.10. Accordingly, the faulted section is identified and its section is isolated. Then, the control is updated, and the restoration action was performed. This action was done by a direct connection to the distribution panel.

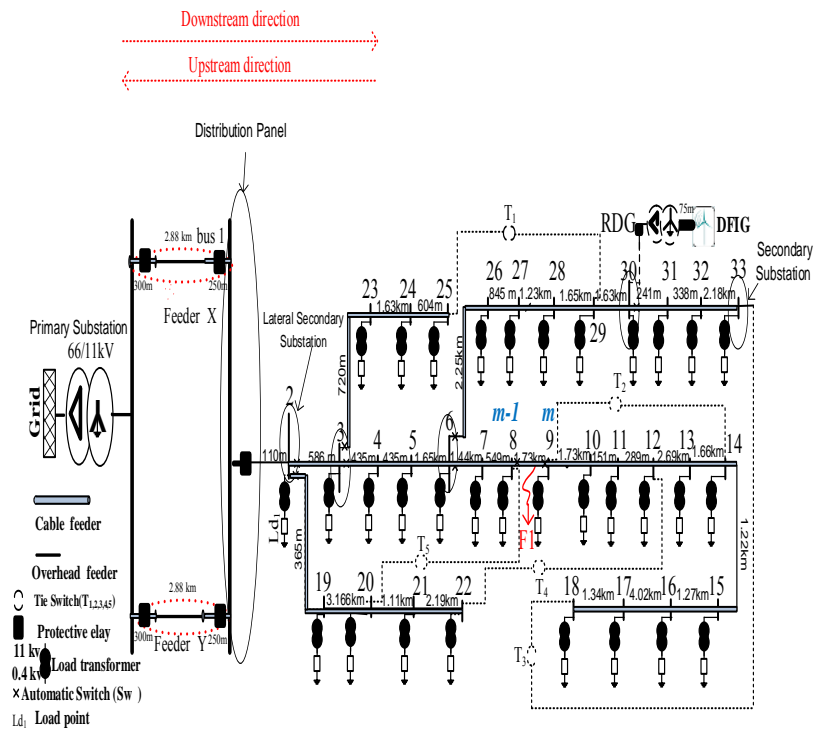

Fig. 11 Single line diagram of the cascaded parallelradial distribution system.

Considering the distribution network shown in Fig. 11 that briefly summarized in the Appendix, a comparison between different controls of fault management and the proposed strategy occurs as follows concerning the communication latency, time delay, and fault management reliability.

\section{Reliability Evaluation of Communication Hops}

6.1 Centralized, decentralized Control $[4,5]$

Based on the centralized and decentralized control rules presented in $[8,9]$, their implementations using both controls needed $m^{2}+7 m+5$ and $2 m+2$ communication hops $N_{c h}$, respectively, when the faulted segment is among substations $m-1$ and $m$. Unfortunately, these communication hops required large latency time.

\subsection{Modified Centralized Control [11]}

The total communications hops' number of this strategy is only five or seven when the faulted section is close or far from the primary station respectively until restoring the service and updating the control center [11]. The total number of communication hops $N_{c h}=n_{t}+6$ where $n_{t}$ is the total number of secondary substation and lateral secondary substation.

\subsection{A proposed Control [10]}

The communication hops' number $N_{c h}$ of modified autonomous control is lower than that of the modified centralized because its hops were not influenced by the feeder size compared with modified centralized which its communications are $(m+4)$. 


\subsection{Discussion}

Table 1. The number of communication hops for proposed control was compared to other control strategies.

\begin{tabular}{|c|c|c|}
\hline Fault Section & Control Strategies & $N_{c h}$ \\
\hline \multirow{4}{*}{$\begin{array}{l}1^{\text {st }} \text { downstream } \\
\text { Primary substation } \\
\text { (Parallel feeder) }\end{array}$} & Centralized [8] & 13 \\
\hline & Decentralized [9] & 4 \\
\hline & Modified Centralized [11] & 7 \\
\hline & A Proposed control [10] & 3 \\
\hline \multirow{4}{*}{$\begin{array}{l}\text { In between two } \\
\text { Lateral secondary } \\
\text { substation } \\
\text { Fault section } \\
(1-2) \\
\end{array}$} & Centralized $[8]$ & 23 \\
\hline & Decentralized [9] & 4 \\
\hline & Modified Centralized [11] & 8 \\
\hline & A Proposed control [10] & 5 \\
\hline \multirow{4}{*}{$\begin{array}{l}1^{\text {st }} \text { downstream } \\
\text { Lateral secondary } \\
\text { substation } \\
\text { Fault section } \\
(2-3) \\
\end{array}$} & Centralized [8] & 35 \\
\hline & Decentralized [9] & 6 \\
\hline & Modified Centralized [11] & 9 \\
\hline & A Proposed control [10] & 6 \\
\hline \multirow{4}{*}{$\begin{array}{l}\text { Fault section } \\
\quad(17-18)\end{array}$} & Centralized [8] & 455 \\
\hline & Decentralized [9] & 36 \\
\hline & Modified Centralized [11] & 24 \\
\hline & A Proposed control [10] & 21 \\
\hline
\end{tabular}

As declares from Table 1, the total number of communication hops $N_{c h}$ for a proposed control is smaller than it's at the centralized, decentralized, and the modified centralized control for $1^{\text {st }}$ downstream Primary substation (Parallel feeder). Despite, the total number of communication hops $N_{c h}$ for a proposed control is larger than the total number of communication hops for the decentralized in fault section (1-2), a proposed control is still smaller than the centralized and modified centralized control. Furthermore, the total number of communication hops for a proposed control is smaller than the centralized, decentralized, and modified centralized control at the fault between two secondary substation Fault sections between buses (17-18) which consider the far secondary substation for the feeder. To examine a proposed control, this evaluation is extended to all secondary substations in Fig. 11. As seen in Fig. 12, the number of communication hops for a proposed control strategy is smaller than all fault management strategies.

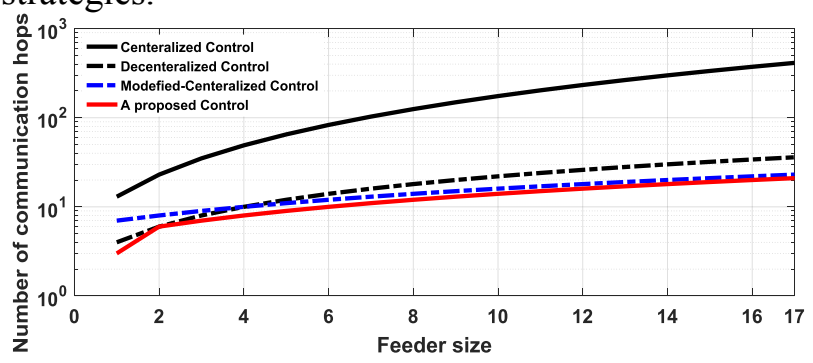

Fig. 12 Impact of feeder size (number of substations) on the communication hops.

\section{Evaluation of Time Delay}

Table 2. Delay Times for Proposed Control Compared to Other Control Strategy.

\begin{tabular}{|c|c|c|}
\hline Fault Section & Control Strategies & \begin{tabular}{|c|} 
Delay \\
Time(s) due \\
to \\
communicat \\
ion hops \\
\end{tabular} \\
\hline \multirow{4}{*}{$\begin{array}{l}1^{\text {st }} \text { downstream } \\
\text { Primary substation } \\
\text { (Parallel feeder) }\end{array}$} & Centralized [8] & 2.1840 \\
\hline & Decentralized [9] & 0.6720 \\
\hline & Modified Centralized [11] & 1.1760 \\
\hline & A Proposed control [10] & 0.504 \\
\hline \multirow{4}{*}{$\begin{array}{c}\text { In between two } \\
\text { Lateral secondary } \\
\text { substation } \\
1-2\end{array}$} & Centralized [8] & 3.8640 \\
\hline & Decentralized [9] & 0.672 \\
\hline & Modified Centralized [11] & 1.3440 \\
\hline & A Proposed control [10] & 0.84 \\
\hline \multirow{4}{*}{$\begin{array}{c}1^{\text {st }} \text { downstream } \\
\text { Lateral secondary } \\
\text { substation } \\
2-3 \\
\end{array}$} & Centralized [8] & 5.88 \\
\hline & Decentralized [9] & 1.008 \\
\hline & Modified Centralized [11] & 1.5120 \\
\hline & A Proposed control [10] & 1.08 \\
\hline \multirow{4}{*}{$17-18$} & Centralized [8] & 76.44 \\
\hline & Decentralized [9] & 6.048 \\
\hline & Modified Centralized [11] & 4.032 \\
\hline & A Proposed control [10] & 3.528 \\
\hline
\end{tabular}

Its response time was calculated based on communication latency for smart grids in [90], where it informs that the communication latency is a random quantity. Thus, it was modeled by utilizing the function of probability density with time mean value of $\left(\Delta_{t}=0.168 \mathrm{~s}\right)$ for radio communication among the substation agents. Accordingly, the response time was calculated by $\left(N_{c h} * \Delta_{t}\right)$. Based on the result from Table 2, the response times for proposed fault management is a small delay time compares with modified centralized control for first, third, and four cases. As declares from the same table, the same delay times for a proposed control strategy in the second case compared with decentralized control. This result is caused by the near-fault section.

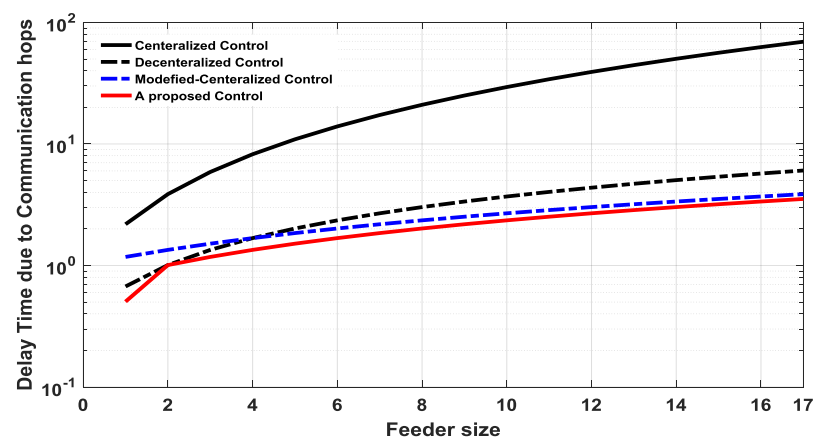

Fig. 13 Impact of feeder size (number of substations) on the delay times due to communication hops.

Similarly, in Fig.13, a proposed control gives a small delay time when using different faults in between all secondary substations in the selected system. 


\section{Evaluation of Fault Management Reliability}

Based on the overall fault management reliability Ro rules presented in [10] and [11]. Its reliability calculates by multiplying the two types of reliability. Firstly, the reliability of fault detection $D_{m}$ in centralized, decentralized, and a proposed control calculated based on the failure index for FPI or the reliability of fault location $D_{\mathrm{m}}$ in modified centralized calculates based on another statistical study for the fault-locator algorithm's average error $f_{\mathrm{n}}$ related to each segment as a ratio of the section length. All fault location functions have reliability $D_{m}$ estimated by simulating the fault cases that occurred in the smart distribution grid shown in Fig. 11 using the ATP-EMTP program and then evaluating their performance using MATLAB code.

For different fault management strategies, the reliability of fault detection is calculated as follow:

\subsection{Centralized, decentralized Control}

The fault detection reliability $D_{m}$ of fault indicators is computed as a function of fault indicator $(h)$.

$$
D_{m}=\prod_{s=1}^{m}(1-h)
$$

where $h=$ the failure index of fault indicator $(h=0.001)$

Based on the number of communication hops $\left(N_{c h}\right)$ its reliability can be calculated. Firstly, for centralized control

$$
R_{m}=\prod_{s=1}^{m^{2}+7 m+5}(1-f)
$$

where $s=$ the sliding index

$$
\mathrm{f}=\text { communication index }(f=0.001) \text {. }
$$

The reliability of communication hops for decentralized control strategy as follow:

$$
R_{m}=\prod_{s=1}^{2 m+2}(1-f)
$$

The overall fault section identification reliability Ro is:

$$
R_{o}=R_{m} \times D_{m}
$$

\subsection{Modified Centralized Control}

The reliability of the fault location is using for a modified centralized control strategy.

$$
D_{m}=\prod_{s=1}^{m}(1-e)
$$

where $h=$ the failure index of fault locator average

\begin{tabular}{|c|c|c|c|c|}
\hline $\begin{array}{c}\text { Fault } \\
\text { Section }\end{array}$ & Control Strategies & $\boldsymbol{R}_{m}$ & $D_{m}$ & $\boldsymbol{R}_{o}$ \\
\hline \multirow{4}{*}{$\begin{array}{c}1^{\text {st }} \\
\text { downstream } \\
\text { Primary } \\
\text { substation } \\
\text { (Parallel } \\
\text { feeder) }\end{array}$} & Centralized [8] & 0.987 & 0.999 & 0.986 \\
\hline & Decentralized [9] & 0.996 & 0.999 & 0.995 \\
\hline & Modified Centralized [11] & 0.993 & 0.966 & 0.9596 \\
\hline & A Proposed control [10] & 0.997 & 0.998 & 0.995 \\
\hline \multirow{4}{*}{$\begin{array}{c}\text { In between } \\
\text { two } \\
\text { Lateral } \\
\text { secondary } \\
\text { substation } \\
1-2\end{array}$} & Centralized [8] & 0.977 & 0.998 & 0.975 \\
\hline & Decentralized [9] & 0.996 & 0.999 & 0.995 \\
\hline & Modified Centralized [11] & 0.992 & 0.966 & 0.9586 \\
\hline & A Proposed control [10] & 0.995 & 0.998 & 0.993 \\
\hline \multirow{4}{*}{$\begin{array}{c}1^{\text {st }} \\
\text { downstream } \\
\text { Lateral } \\
\text { secondary } \\
\text { substation } \\
2-3\end{array}$} & Centralized [8] & 0.965 & 0.997 & 0.962 \\
\hline & Decentralized [9] & 0.994 & 0.998 & 0.992 \\
\hline & Modified Centralized [11] & 0.991 & 0.950 & 0.9417 \\
\hline & A Proposed control [10] & 0.995 & 0.998 & 0.993 \\
\hline \multirow{4}{*}{$17-18$} & Centralized [8] & 0.634 & 0.982 & 0.6229 \\
\hline & Decentralized [9] & 0.964 & 0.983 & 0.9483 \\
\hline & Modified Centralized [11] & 0.976 & 0.841 & 0.8214 \\
\hline & A Proposed control [10] & 0.995 & 0.998 & 0.993 \\
\hline
\end{tabular}
error $(e)$

The reliability of communication hops for modified centralized control strategy as follow:
Table 3 The Performance of Overall Reliability at Different Fault Management Strategies.

$$
R_{m}=\prod_{s=1}^{n_{t}+6}\left(1-f_{n}\right)
$$

\subsection{A proposed Control}

The fault detection reliability $D_{\mathrm{m}}$ for each fault indication failure is $h$ is computed as;

$D_{m}=\left(1-h_{m-1}\right) \times\left(1-h_{m}\right)=(1-h)^{2}$

The reliability of communicated messages affects the decision of fault section identification is computed as $(1-f)^{2}$ whatever the network size and then $(1-f)^{3}$ for reclosing and updating the control center. Therefore, the total reliability of communication hops used is typically $(1-f)^{5}$ at all secondary substation except when the fault in the first section is reduced to $(1-f)^{3}$.

Based on the results in Table 3, the overall reliability of a proposed control strategy gives the best value (large value) as compares with modified centralized control for first, second, and third cases. Furthermore, the reliability of the proposed control attains a better value compares with different fault management strategies at the far secondary substation fault section (17-18). 


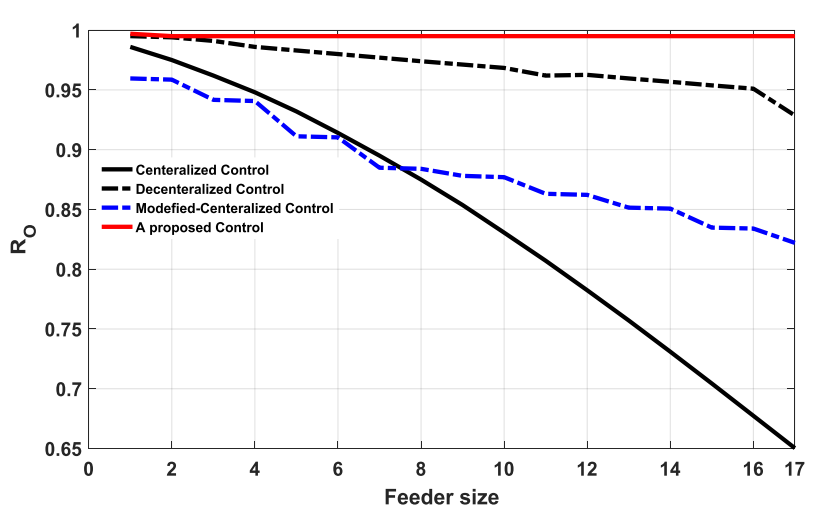

Fig. 14 Impact of feeder size ( number of substations) on the overall reliability.

Despite the reliability of a proposed control gives a small value at the first secondary substation compares with different fault management strategies seen in Fig. 14. The reliability for proposed control gives a fixed value for all secondary substations after near substations such as first, and second cases. The result declares its superiority, the efficacy of a proposed concept compares with centralized, and modified centralized. Consequently, a proposed fault management process uses to reduce the outage times for the customers where this consequently reduces the risk of distribution network component damages. Accordingly, a proposed system with the aid of autonomous control can be examined by calculating the failure probability of its management in the next section.

\section{Evaluation of Failure Probability}

The ratio for failure probability function of different fault management strategies is calculated as follow: $P(f)=\left(1-R_{o}\right) \times 100$

Based on (7), the last evaluation in this paper was done. As declares in Table (4), the failure probability ratio of a proposed control gives a smaller than all fault management.

Furthermore, the failure probability ratio extends to all secondary substation seen in Fig. 15. As seen in this figure, a proposed control attains a small failure probability ratio at all secondary substations.

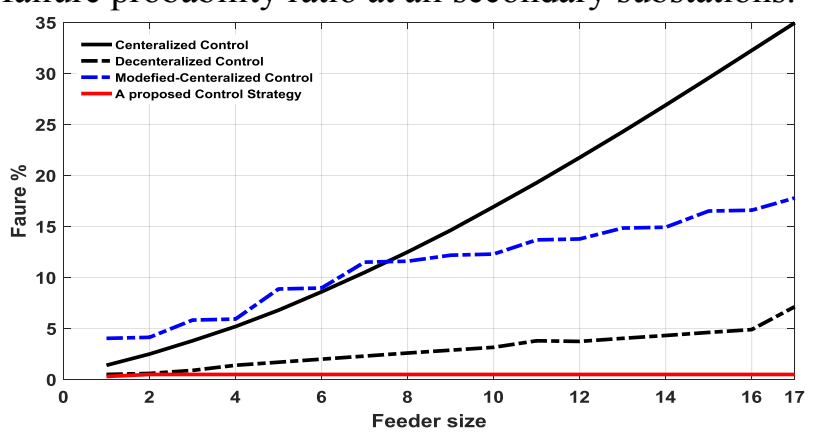

Fig. 15 Impact of feeder size (number of substations) on the failure probability.
Table 4 Failure Probability Ratio at Different Fault Management Strategies.

\begin{tabular}{|c|c|c|}
\hline Fault Section & Control Strategies & $P(f) \%$ \\
\hline \multirow{4}{*}{$\begin{array}{l}1^{\text {st }} \text { downstream } \\
\text { Primary substation } \\
\text { (Parallel feeder) }\end{array}$} & Cen & 1.40 \\
\hline & Decentralized [9] & 0.50 \\
\hline & Modified Centralized [11] & 4.04 \\
\hline & A Proposed control [10] & 0.50 \\
\hline \multirow{4}{*}{$\begin{array}{c}\text { In between two } \\
\text { Lateral secondary } \\
\text { substation } \\
1-2\end{array}$} & Centralized $[8]$ & 2.5 \\
\hline & \begin{tabular}{|l} 
Dece \\
\end{tabular} & 0.5 \\
\hline & Modified Centr & 4.14 \\
\hline & A Proposed control [10] & 0.50 \\
\hline \multirow{4}{*}{$\begin{array}{c}1^{\text {st }} \text { downstream } \\
\text { Lateral secondary } \\
\text { substation } \\
2-3 \\
\end{array}$} & Centralized [8] & 3.80 \\
\hline & alized [9] & 0.8 \\
\hline & Modified Centı & 5.83 \\
\hline & A Prop & 0.6 \\
\hline \multirow{4}{*}{$17-18$} & Centralized [8] & 37.71 \\
\hline & Decentralized [9] & 5.17 \\
\hline & Modified Centralized [11] & 17.86 \\
\hline & A Proposed control [10] & 0.6 \\
\hline
\end{tabular}

\section{Conclusions}

In this paper, a proposed control strategy with the aid of autonomous control was introduced. Accordingly, the communication hops number for accomplishing the fault management were reduced. Its performance was evaluated and compared with other published control systems. Furthermore, a proposed strategy had a small probability of system unreliability compared with centralized, decentralized, and modified centralized control. The discussions have shown the superiority of a proposed control strategy with its fast and reliable performance as compares with other fault management strategies.

\section{Appendix}

A practical example from the Egyptian distribution (33 bus system) was considered as a testing example (sample example from all network) as shown in Fig. 11 with a cascaded parallel-radial distribution system. In this paper, the Egyptian distribution network has been selected in this study. The radial feeders are linked with normally automatic closed switches (SW) and normally open tie switches $\left(\mathrm{T}_{1,2,3,4,5}\right)$ to facilitate the faulty section isolation and the restoration process. These selected points are based on the power flow of feeders. The system consists of two overhead parallel incoming feeders, cascaded with one outgoing cable feeder. The buses are renumbered for simplicity, 64 normally closed switches located at each section terminals while one circuit breaker is added at the beginning of the feeder. Also, normally open switches (Tie switches) are added to the system to verify system reconfiguration. Each of the overhead parallel incoming feeder parameters is illustrated in Table 5. 
This outgoing feeder is characterized by the positive sequence data of the feeder and loads listed in Table 6 . The positive and zero sequence resistance are equal to $1.62,0.5(\Omega / \mathrm{km})$, respectively, while the positive and zero sequences inductance are equal to $3.82,7.14(\mathrm{mH} / \mathrm{km})$, respectively, the positive and zero sequence capacitances are equal to 0.0067 and $0.0041 \mu \mathrm{f} / \mathrm{km}$, respectively. Also, wind turbine data are introduced in Table 7.

Table 5 Parameters of Incoming Feeders.

\begin{tabular}{|c|c|c|c|}
\hline$\underset{1}{\text { Symbo }}$ & Quantity & Overhead & Unit \\
\hline$R_{l}$ & $\begin{array}{l}\text { Positive sequence } \\
\text { resistance }\end{array}$ & 0.194 & $(\Omega / \mathrm{km})$ \\
\hline$R_{0}$ & $\begin{array}{l}\text { Zero sequence } \\
\text { resistance }\end{array}$ & 0.3 & $(\Omega / \mathrm{km})$ \\
\hline$L_{1}$ & $\begin{array}{l}\text { Positive sequence } \\
\text { inductance }\end{array}$ & 1.78 & $(\mathrm{mH} / \mathrm{km})$ \\
\hline$L_{0}$ & $\begin{array}{l}\text { zero sequence } \\
\text { inductance }\end{array}$ & 6.1 & $(\mathrm{mH} / \mathrm{km})$ \\
\hline$C_{l}$ & $\begin{array}{l}\text { Positive sequence } \\
\text { capacitance }\end{array}$ & 0.015 & $(\mu \mathrm{F} / \mathrm{km})$ \\
\hline $\mathrm{C}_{0}$ & $\begin{array}{l}\text { zero sequence } \\
\text { capacitance }\end{array}$ & 0.0049 & $(\mu \mathrm{F} / \mathrm{km})$ \\
\hline
\end{tabular}

Table 6 Parameters for the Outgoing Feeder.

\begin{tabular}{|c|c|c|c|c|}
\hline \hline & & & & \\
\multirow{2}{*}{ Bus number } & & & & \\
& \hline \hline & Line impedance & \multicolumn{2}{c|}{ Load Power } \\
\hline 2 & 0.0922 & 0.0470 & 100.0 & 60.0 \\
3 & 0.4930 & 0.2511 & 90.0 & 40.0 \\
4 & 0.3660 & 0.1864 & 120.0 & 80.0 \\
5 & 0.3811 & 0.1941 & 60.0 & 30.0 \\
6 & 0.8190 & 0.7070 & 60.0 & 20.0 \\
7 & 0.1872 & 0.6188 & 200.0 & 100.0 \\
8 & 0.7114 & 0.2351 & 200.0 & 100.0 \\
9 & 1.0300 & 0.7400 & 60.0 & 20.0 \\
10 & 1.0040 & 0.7400 & 60.0 & 20.0 \\
11 & 0.1996 & 0.0650 & 45.0 & 30.0 \\
12 & 0.3744 & 0.1238 & 60.0 & 35.0 \\
13 & 1.4680 & 1.1550 & 60.0 & 35.0 \\
14 & 0.5416 & 0.7129 & 120.0 & 80.0 \\
15 & 0.5910 & 0.5260 & 60.0 & 10.0 \\
16 & 0.7463 & 0.5450 & 60.0 & 20.0 \\
17 & 1.2890 & 1.7210 & 60.0 & 20.0 \\
18 & 0.7320 & 0.5740 & 90.0 & 40.0 \\
19 & 0.1640 & 0.1565 & 90.0 & 40.0 \\
20 & 1.5042 & 1.3554 & 90.0 & 40.0 \\
21 & 0.4095 & 0.4784 & 90.0 & 40.0 \\
22 & 0.7089 & 0.9373 & 90.0 & 40.0 \\
23 & 0.4512 & 0.3083 & 90.0 & 50.0 \\
24 & 0.8980 & 0.7091 & 420.0 & 200.0 \\
25 & 0.8960 & 0.7011 & 420.0 & 200.0 \\
26 & 0.2030 & 0.1034 & 60.0 & 25.0 \\
27 & 0.2842 & 0.1447 & 60.0 & 25.0 \\
28 & 1.0590 & 0.9337 & 60.0 & 20.0 \\
29 & 0.8042 & 0.7006 & 120.0 & 70.0 \\
30 & 0.5075 & 0.2585 & 200.0 & 600.0 \\
31 & 0.9744 & 0.9630 & 150.0 & 70.0 \\
32 & 0.3105 & 0.3619 & 210.0 & 100.0 \\
33 & 0.3410 & 0.5302 & 60.0 & 40.0 \\
\hline \hline
\end{tabular}

Table 7 Selected Wind Turbine Generator Data

\begin{tabular}{|l|r|r|r|}
\hline Parameter & \multicolumn{1}{|l|}{ Value } & Parameter & Value \\
\hline Rated power & $3 \times 0.45 \mathrm{MW}$ & Stator reactance & $0.18 \mathrm{p} . u$. \\
Stator rated voltage & $0.575 \mathrm{kV}$ & Rotor reactance & $0.16 \mathrm{p} . u$. \\
Stator resistance & 0.023 p.u. & dc-link voltage & $1.15 \mathrm{kV}$ \\
Rotor resistance & 0.016 p.u. & dc-link capacitor & $10 \mathrm{mF}$ \\
\hline
\end{tabular}

\section{References:}

[1] G. Xiang, A. Xin, The application of self-healing technology in smart grid, in: Power and Energy Engineering Conference (APPEEC), AsiaPacific, March 25-28, 2011.

[2]A. Zidan, M. Khairalla, A. M. Abdrabou, T. Khalifa, K. Shaban, A. Abdrabou, R El-Shatshat, and A. Gaouda, Fault Detection, Isolation, and Service Restoration in Distribution Systems: State-of-the-Art and Future Trends, IEEE Transactions on Smart Grid, vol. 8, no 5, pp. 2170-2185, Sep.2017.

[3] N. F. Sherbilla, M. A. Elsadd, T. A. Kawady, N. I. Elkalashy and A. I. Taalab, Autonomous Backup Selectivity Technique for Line-toGround Faults in Automated Distribution Networks, $21^{\text {st }}$ International Middle East Power Systems Conference (MEPCON), Cairo, Egypt, pp. 952-957, 2019.

[4]O. Abood, M. Elsadd, and S. Guirguis, Investigation of cryptography algorithms used for security and privacy protection in smart grid, Nineteenth International Middle East Power Systems Conference (MEPCON), December 2017.

[5]O. Abood, M. Elsadd, and S. Guirguis, Incorporating Deoxyribonucleic Acid in AES Scheme for Enhancing Security and Privacy Protection, Journal of Theoretical and Applied Information Technology, vol. 97. no. 02, pp. 349360, January 2019.

[6] L. S. Khalifa, M. A. Elsadd, R. A. Abd El-Aal, and S. M. El-Makkawy, Enhancing Recloser-Fuse Coordination Using Distributed Agents in Deregulated Distribution Systems, Twentieth International Middle East Power Systems Conference (MEPCON), Cairo, Egypt, pp. 948955, , 2018.doi: 10.1109/MEPCON.2018.8635116

[7]C.xia, and B. Liu, Hierarchical management and control based on MAS for distribution grid via intelligent mode switching, Elect Power and Energy Syst., vol. 54,2014, pp. 352-366.

[8] M. Nordman and M. Lehtonen, an agent concept for managing electrical distribution networks, In IEEE Trans Power Delivery, V.20, No. 2, 2005, pp.696-703.

[9] G. Zhabelova, V. Vyatkin, Multiagent smart grid automation architecture based on IEC61850/61499 
intelligent logical nodes, IEEE Trans. Ind. Electron, V.59, 2012, pp.2351-2362.

[10] Tarhuni, N. Elkalashy, T. Kawady, M. Lehtonen, Autonomous Control Strategy for Fault Management in Distribution Networks, In Journal of Elect. Power Syst. Res, V.121, 2015, pp.252259.

[11] M. A. Elsadd, N. I. Elkalashy, T. A. Kawady, A.M. I. Taalab, and Matti Lehtonen, Incorporating Earth Fault Location in Management Control Scheme for Distribution Networks, In IET Gen Transm Distrib, V.10, No. 10, 2016, pp. 23892398.

[12] M. Pipattanasomporn, H. Feroze, and S. Rahman, Multi agent systems in a distributed smart grid: design and implementation, in: Proc. IEEE Power Systems Conference and Exposition, March, 2009, pp.1-8.

[13] U.S. Department of Energy Office of Energy Efficiency and Renewable Energy, The Smart Grid: An Introduction, prepared by Litos Strategic Communication, available online:

http://energy.gov/sites/prod/files/oeprod/Documents andMedia/DOE SG Book Single Pages(1).pdf.

[14] U.S. Department of Energy, The potential benefits of distributed generation and rate-related issues that may impede its expansion, in: A Study Pursuant to Section 1817 of the Energy Policy Act of 2005, U.S. Department of Energy, June 2007.

[15] H.B. Puttgen, P.R. MacGregor, F.C. Lambert, Distributed generation: semantic hype or the dawn of a new era?, IEEE Power Energy Mag., V.1, No. 1, 2003, pp.22-29.

[16] M. Amin, B.F. Wollenberg, Toward a smart grid: power delivery for the $21^{\text {st }}$ century, IEEE Power Energy Mag., V. 3, No. 5, 2005, pp. 34-41.

[17] K. Moslehi, R. Kumar, Vision for a self-healing power grid, in: ABB Review, No.4, 2006.

[18] Farhan H. Malik, Matti Lehtonen, A review: Agents in smart grids, Electric Power Systems Research., V. 131, 2016, pp.71-79.

[19] National Instruments project on, Smart Grid Evolution: A New Generation of Intelligent Electronic Devices,

Available online:

http://www.renewableenergyworld.com/articles/201 4/03/smart-grid-evolution-a-newgeneration-ofintelligent-electronic-devices.html.

[20] Siemens report on, Smart Grids and Energy Storage: A Fluctuating Balance, available online: http://www.siemens.com/innovation/en/home/pic turesof-the-future/energy-and-efficiency/smartgrids-and-energy-storageinternet-of-energy.html.
[21] P.S. Miriam, E.R. Roger, Power Line Carrier Channel \& Application Considerations for Transmission Line Relaying, Pulsar Technologies, document number: C045-P0597.

[22] F. Aalamifar, H.S. Hassanein, G. Takahara, Viability of power line communication for the smart grid, in: Proc. IEEE Communications (QBSC), 26 $6^{\text {th }}$ Biennial Symposium on, 28-29 May, 2012, pp. 19-23.

[23] D.J. Leeds, The smart grid in 2010: market segments, applications and industry players, GTM Res., July, 2009.

[24] M. Kuzlu, M. Pipattanasomporn, Assessment of communication technologies and network requirements for different smart grid applications, Innovative Smart Grid Technologies (ISGT) IEEE PES, 24-27 Feb,2013, pp. 1-6.

[25] J. Ward, N. Vinod, A. Visvakumar, K. Babak, K. Mladen, D. Yimai, Communication Requirements and Integration Options for Smart Grid Deployment, Power Systems Engineering Research Center, Wichita State University and Texas A\&M University, (PSERC Publication) 1203, April, 2012.

[26] EPRI, Estimating the costs and benefits of the smart grid, in: Tech. Rep., Electric Power Research Institute, 2011.

[27] D. Castro, J.L. Dutra, The economics of the smart grid, in: Proc. IEEE Communication, Control, and Computing (Allerton), $20114^{\text {th }}$ Annual Allerton Conference on, 28-30 Sept, 2011, pp. 1294-1301.

[28] A.P. Garcia, J. Oliver, D. Gosch, An Intelligent Agent-Based Distributed Architecture for SmartGrid Integrated Network Management, in: Local Computer Networks (LCN), 2010 IEEE 35 Conference on, October, 2010, pp.1013-1018.

[29] M.K. Kouluri, R.K. Pandey, Intelligent agent based micro grid control, in: Proc. IEEE Intelligent Agent and Multi-Agent Systems (IAMA), $20112^{\text {nd }}$ International Conference on, 79 Sept., 2011, pp. 62-66.

[30] J. Hossack, S.D.J. Mcanhur, J.R. Mcdonald, J. Stokoe, T. Cumming, A multi-agent approach to power system disturbance diagnosis, Proc. Int. Conf. Power Syst. Manage. Control 488 April, 2002, pp.7-322.

[31] S.D.J. McArthur, E.M. Davidson, J.A. Hossack, R. McDonald, Automating power system fault diagnosis through multi-agent system technology, in: Proc. the $37^{\text {th }}$ Hawaii International Conference on System Sciences, 2004, p. 8. 
[32] T. Nagata, H. Sasaki, A multi-agent approach to power system restoration, IEEE Trans. Power Syst. 17 May,2002, pp.457-462.

[33] H.F. Wang, Multi-agent co-ordination for the secondary voltage control in power system contingencies, Proc. IEE Gener. Transm. Distrib. 148, Jan, 2001, pp. 61-66.

[34] D. Bailey, E. Wright, Practical SCADA for Industry, News, Oxford, UK, 2003.

[35] National Communications System, Supervisory Control and Data Acquisition (SCADA) Systems, Technical Information Bulletin 04-1, October 2004.

[36] V. Salehi and O. Mohammed, Developing virtual protection system for control and self-healing of power system, in Proc. IEEE Ind. Appl. Soc. Annu. Meeting, Orlando, FL, USA, Oct. 2011, pp. 1-7.

[37] S. Mohagheghi, J. Stoupis, and Z. Wang, Communication protocols and networks for power systems-current status and future trends, in Proc. IEEE Power Syst. Conf. Expo., Seattle, WA, USA, Mar. 2009, pp. 1-9.

[38] D. Baranovskis and J. Rozenkrons, Integration of the universal earth fault indicator in to the distribution network SCADA system, in Proc. IEEE Russia Power Tech. Conf., St. Petersburg, Russia, Jun. 2005, pp. 1-5.

[39] S. Hodgson, The use GSM and Web based SCADA for monitoring fault passage indicators, in Proc. IEEE Transm. Distrib. Conf. Expo., New Orleans, LA, USA, Apr. 2010, pp. 1-3.

[40] M. A. Shahin, Smart grid self-healing implementation for underground distribution networks, in Proc. IEEE Innov. Smart Grid Technol. Conf., Bengaluru, India, Nov. 2013, pp. 1-5.

[41] E. Coster, W. Kerstens, and T. Berry, Selfhealing distribution networks using smart controllers, in Proc. 22 ${ }^{\text {nd }}$ Int. Conf. Exhibit. Elect. Distrib. (CIRED), Stockholm, Sweden, Jun. 2013, pp. 1-4.

[42] A. Varžiû, K. Slivariû, and M. Šporec, detecting faults in MV network using GPRS, in Proc. $22^{\text {nd }}$ Int. Conf. Elect. Distrib., Stockholm, Sweden, Jun. 2013, pp. 1-3.

[43] R. Jenkins and D. Dolezilek, Case study: Using IEC 61850 methods for RTU replacement and distributed automation, in Proc. $10^{\text {th }}$ Annu. Western Power Del. Autom. Conf., Spokane, WA, USA, Apr. 2008, pp. 1-17.

[44] D. T. Brown and A. L. Gielink, A Utility's Experience in the Implementation of Substation Automation Projects
[Online]. Available:

https://www.gedigitalenergy.com/smartgrid/Dec07/

$\underline{5}$ substation_automation.pdf, Feb, 2015.

[45] N. Vlassis, A concise introduction to multiagent systems and distributed artificial intelligence, Synth. Lectures Artif. Intell. Mach.Learn., Vol. 1, No. 1, 2007, pp. 1-71.

[46] S. R. Islam, K. M. Muttaqi, and D. Sutanto, A decentralized multiagent-based voltage control for catastrophic disturbances in a power system, IEEE Trans. Ind. Appl., Vol. 51, No. 2, Mar./Apr. 2015, pp. 1201-1214.

[47] X. Tong et al., The study of a regional decentralized peer-to-peer negotiation-based wide-area backup protection multi-agent system, IEEE Trans. Smart Grid, Vol. 4, No. 2, Jun. 2013, pp. 1197-1206.

[48] M. Shafie-Khah and J. P. S. Catalao, "A stochastic multi-layer agent-based model to study electricity market participants behaviour, IEEE Trans. Power Syst., Vol. 30, No. 2, Mar. 2015, pp. 867-881.

[49] S. D. J. McArthur et al., Multi-agent systems for power engineering applications-Part I: Concepts, approaches, and technical challenges, IEEE Trans. Power Syst., Vol. 22, No. 4, Nov. 2007, pp. 1743-1752.

[50] S. D. J. McArthur et al., Multi-agent systems for power engineering applications-Part II: Technologies, standards, and tools for building multi-agent systems, IEEE Trans. Power Syst., Vol. 22, No. 4, Nov. 2007, pp. 1753-1759.

[51] A. Zidan and E. F. El-Saadany, A cooperative multiagent framework for self-healing mechanisms in distribution systems, IEEE Trans.Smart Grid, Vol. 3, No. 3, Sep. 2012, pp. 1525-1539.

[52] M.-S. Tsai and Y.-T. Pan, Application of BDIbased intelligent multiagent systems for distribution system service restoration planning, Eur.Trans. Elect. Power, Vol. 21, No. 5, Jul. 2011, pp. 1783-1801.

[53] T. Nagata and H. Sasaki, A multi-agent approach to power system restoration, IEEE Trans. Power Syst., Vol. 17, No. 2, May 2002, pp. 457-462.

[54] Y. Xu and W. Liu, Novel multiagent based load restoration algorithm for microgrids, IEEE Trans. Smart Grid, Vol. 2, No. 1, Mar. 2011, pp. 152-161.

[55] J. M. Solanki, S. Khushalani, and N. N. Schulz, A multi-agent solution to distribution systems restoration, IEEE Trans. Power Syst., Vol. 22, No. 3, Aug. 2007, pp. 1026-1034. 
[56] I. S. Baxevanos and D. P. Labridis, Implementing multiagent systems technology for power distribution network control and protection management, IEEE Trans. Power Del., Vol. 22, No. 1, Jan. 2007, pp.433-443.

[57] C. P. Nguyen and A. J. Flueck, Agent based restoration with distributed energy storage support in smart grids, IEEE Trans. Smart Grid, Vol. 3, No. 2, Jun. 2012, pp.1029-1038.

[58] Z. Wang and J. Wang, Self-healing resilient distribution systems based on sectionalization into microgrids, IEEE Trans. Power Syst., Vol. 30, No. 6, Nov. 2015, pp. 3139-3149.

[59] M. Eriksson, M. Armendariz, O. O. Vasilenko, A. Saleem, and L. Nordstrom, Multi-agent-based distribution automation solution for self-healing grids, IEEE Trans. Ind. Electron., Vol. 62, No. 4, Apr. 2015, pp. 2620-2628.

[60] M. Shahidehpour, Y. Wang, Communication and Control in Electric Power Systems: Applications of Parallel and Distributed Processing, John Wiley \& Sons, New Jersey, Ch. 1 \& Ch. 10,2003, pp. 349-389.

[61] T. Ghanbari and E. Farjah, A multiagent-based fault-current limiting scheme for the microgrids, IEEE Trans. Power Del., Vol. 29, No. 2, Apr. 2014, pp. 525-533.

[62] M. Kezunovic, Smart fault location for smart grids" IEEE Trans. Smart Grid, Vol. 2, No. 1, Mar. 2011, pp. 11-22.

[63] G. D. Ferreira et al., Impedance-based fault location for overhead and underground distribution systems, in Proc. North Amer. Power Symp. Champaign, IL, USA, Sep. 2012, pp. 1-6.

[64] M.-S. Choi, S.-J. Lee, S.-I. Lim, D.-S. Lee, and $\mathrm{X}$. Yang, A direct three-phase circuit analysisbased fault location for line-to-line fault, IEEE Trans. Power Del., Vol. 22, No. 4, Oct. 2007, pp. 2541-2547.

[65] M. Elsadd, N. Elkalashy, T. Kawady, and A. Taalab, Earth fault location determination independent of fault impedance for distribution networks, in International Transactions on Electrical Energy Systems, Vol. 27, No 5, 5 May 2017,pp. 2389-2398.

[66] S. Lin, Z. Y. He, X. P. Li, and Q. Q. Qian, Travelling wave time-frequency characteristicbased fault location method for transmission lines, IET Gener., Transm. Distrib., Vol. 6, No. 8, Aug. 2012, pp. 764-772.

[67] M. Pourahmadi-Nakhli and A. A. Safavi, Path characteristic frequency-based fault locating in radial distribution systems using wavelets and neural networks, IEEE Trans. Power Del., Vol. 26, No. 2, Apr. 2011, pp. 772-781.

[68] J. Sadeh, E. Bakhshizadeh, and R. Kazemzadeh, A new fault location algorithm for radial distribution systems using modal analysis, Int. $J$. Elect. Power Energy Syst., Vol. 45, No. 1, Feb. 2013, pp. 271-278.

[69] J. C. S. Souza, M. A. P. Rodrigues, M. T. Schilling, and M. B. D. C. Filho, Fault location in electrical power systems using intelligent systems techniques, IEEE Trans. Power Del., Vol. 16, No. 1, Jan. 2001, pp. 59-67.

[70] C. Y. Teo and H. B. Gooi, Artificial intelligence in diagnosis and supply restoration for a distribution network, IEE Proc. Gener. Transm. Distrib., Vol. 145, No. 4, Jul. 1998, pp. 444-450.

[71] J. A. Momoh, L. G. Dias, and D. N. Laird, An implementation of a hybrid intelligent tool for distribution system fault diagnosis, IEEE Trans. Power Del., Vol. 12, No. 2, Apr. 1997, pp. 10351040.

[72] F. Mekic, K. Alloway, C. Angelo, and R. Goodin, Fault detection isolation and restoration on the feeder (FDIR): Pick your technology, in Proc. $21^{\text {st }}$ Int. Conf. Elect. Distrib., Frankfurt, Germany, Jun. 2011, pp.1-8.

[73] R. Wernsing et al., Implementation of an innovative advanced loop scheme to provide distribution reliability improvement, in Proc. IEEE Rural Elect. Power Conf., Orlando, FL, USA, May 2010, pp. B2-B2-8.

[74] S. Rusitschka, K. Eger, and C. Gerdes, Smart grid data cloud: A model for utilizing cloud computing in the smart grid domain, in Proc. $1^{\text {st }}$ IEEE Int. Conf. Smart Grid Commun., Gaithersburg, MD, USA, Oct. 2010, pp. 483488.

[75] L. Whei-Min and C. Hong-Chan, A new approach for distribution feeder reconfiguration for loss reduction and service restoration, IEEETrans. Power Del., Vol. 13, No. 3, Jul. 1998, pp.870-875.

[76] M. R. Kleinberg, K. Miu, and H.-D. Chiang, Improving service restoration of power distribution systems through load curtailment of in-service customers, IEEE Trans. Power Syst., Vol. 26, No. 3,Aug. 2011, pp.1110-1117.

[77] D. Shirmohammadi, Service restoration in distribution networks via network reconfiguration, IEEE Trans. Power Del., Vol. 7, No. 2, Apr. 1992, pp. 952-958. 
[78] M.-S. Tsai, Development of an object-oriented service restoration expert system with load variations, IEEE Trans. Power Syst., Vol. 23, No. 1, Feb. 2008, pp. 219-225.

[79] S. Srivastava and K. L. Butler-Burry, Expertsystem method for automatic reconfiguration for restoration of shipboard power systems, IEE Proc. Gener., Transm. Distrib., Vol. 153, No. 3, May 2006, pp. 253-260.

[80] S. J. Lee, K. H. Kim, H. Y. Kim, J. K. Lee, and K. Y. Nam, Expert system-aided service restoration in distribution automation, in Proc. IEEE Int. Conf. Syst. Man Cybern., Vol. 1. Chicago, IL, USA,Oct. 1992, pp. 157-161.

[81] C.-C. Liu, S. J. Lee, and S. S. Venkata, An expert system operational aid for restoration and loss reduction of distribution systems, IEEE Trans. Power Syst., Vol. 3, No. 2, May 1988, pp. 619-626.

[82] K. Manjunath and M. R. Mohan, A new hybrid multi-objective quick service restoration technique for electric power distribution systems, Int. J. Elect. Power Energy Syst., Vol. 29, No. 1, Jan. 2007, pp. 51-64.

[83] Y. Kumar, B. Das, and J. Sharma, Multi objective, multi constraint service restoration of electric power distribution system with priority customers, IEEE Trans. Power Del., Vol. 23, No. 1, Jan. 2008, pp. 261-270.

[84] Y. Jiang, J. Jiang, and Y. Zhang, A novel fuzzy multi objective model using adaptive genetic algorithm based on cloud theory for service restoration of shipboard power systems, IEEE Trans. Power Syst., Vol. 27, No. 2, May 2012, pp. 612-620.

[85] S. Khushalani, J. M. Solanki, and N. N. Schulz, Optimized restoration of unbalanced distribution systems, IEEE Trans. Power Syst., Vol. 22, No. 2, May 2007, pp. 624-630.

[86] R. M. Ciric and D. S. Popovic, Multi-objective distribution network restoration using heuristic approach and mix integer programming method, Int. J. Elect. Power Energy Syst., Vol. 22, No.7, Oct. 2000, pp. 497-505.

[87] M.-S. Tsai, and Y.-T. Pan, Application of BDIbased intelligent multiagent systems for distribution system service restoration planning, Eur.Trans. Elect. Power, Vol. 21, No. 5, Jul. 2011, pp. 1783-1801.

[88] T. Nagata, and H. Sasaki, A multi-agent approach to power system restoration, IEEE Trans. Power Syst., Vol. 17, No. 2, May 2002, pp. 457-462.

[89] Z. Wang and J. Wang, Self-healing resilient distribution systems based on sectionalization into microgrids, IEEE Trans. Power Syst., Vol. 30, No. 6, Nov. 2015, pp. 3139-3149.

[90] C. Nguyen, A. Flueck, Modeling of communication latency in smart grid, in IEEE Power and Energy Society General Meeting Detroit, MI, USA, July 2011, pp. 24-29.

\section{Creative Commons Attribution License 4.0} (Attribution 4.0 International, CC BY 4.0)

This article is published under the terms of the Creative Commons Attribution License 4.0 https://creativecommons.org/licenses/by/4.0/deed.en US 